\title{
SENSOR NETWORK PERIODICAL DATA ASSEMBLY USING IMBALANCED CLUSTERING ENERGY EFFICIENT MECHANISM
}

\author{
${ }^{1}$ Munusamy, K. and ${ }^{2}$ R.M.S. Parvathi \\ ${ }^{1}$ Bharathiar University, Coimbatore, TamilNadu, India \\ ${ }^{2}$ Department of Computer science and Engineering, \\ Anna University, Sengunthar College of Engineering Tiruchengode, TamilNadu, India
}

Received 2014-02-08; Revised 2014-02-24; Accepted 2014-06-28

\begin{abstract}
Fast technical advances and development in wireless communication resulted in the huge consumption of wireless sensor networks. The nodes in these networks are ready to be sensed, processing the data and transmitting radio units. While several research works have been conducted related to the power-aware and energy-efficiency using Cooperative Networking protocol (CONET) that reforms clusters but the multiple rate remains to be explored. Due to the huge constraint in energy, the resources of sensor networks should be aimed at increasing the lifetime of sensors. This motivates us to seek a different way for severe energy constraint to overcome the limitations of the previous works by utilizing periodical data assembly in which energy efficiency and network scalability is achieved for inter-cluster communication using imbalanced clustering and multi hop routing. In this approach, ICEE focuses on two main strategies, attain efficiency in energy and increase scalability of network by making a cluster head choose a relay node accordingly to its residual energy and distance to base station. To start with first the focus is made on Imbalanced Clustering Energy Efficient (ICEE) mechanism for periodical data assembly where cluster heads are elected by localized competition in wireless sensor networks. The ICEE partitions enables the nodes into clusters of imbalanced size and clusters closer to the base station have lesser sizes than those farther away from the base station. The cluster heads nearer to the base station keep some energy for the data forwarding during inter-cluster. The implications of the research reveals that Imbalanced Cluster Energy Efficient mechanism ensures inter-cluster communication with the help of energy-aware multi hop routing protocol and not only decide on correct power level but also help to create clusters of imbalanced size. Numerical results measured in terms of communication energy, power consumption and cluster head probability ratio in wireless sensor network depict superior performance in comparison with CONET using ns 2 simulator.
\end{abstract}

Keywords: Multiple Rate, Imbalanced Clustering Energy-Efficient Mechanism, Cooperative Networking Protocol, Distributed Competitive Algorithm, Multi Hop Routing, Cluster Head Probability

\section{INTRODUCTION}

Widespread research on energy aware routing is being conducted, increasing lifetime and survivability in WSN. Organizing sensor networks into clustered architectures is researched over the last few years, resulting to the larger number of task specific clustering protocols. Clustering is one such approaches for energy efficient scalable scattered sensor networks.
Research concerning multi hop communication between the data source and a data sink is more energy efficient than direct transmission because of the wireless channel individuality. Forwarding schemes are designed when nodes exploit the significance of the individual transmitted messages (Roc1o et al., 2011). Certain researches have concentrated on the unlimited types of problem in a way that the node of relay nature can be positioned anywhere. But there arises certain kinds of

Corresponding Author: Munusamy, K., Bharathiar University, Coimbatore, TamilNadu, India 
physical issues related to the positioning of the relay nodes. To address this issue (Kim et al., 2010) the relay node were positioned at a set of candidate locations.

Recent advances in WSN have resulted in addressing the issues related to sense the environment in a remote manner. In (Kim et al., 2010), problems related to minimizing the delay and ma ximizing the lifetime of nodes in WSN for which events occur infrequently was addressed but waited for longer duration. The increase in the lifetime for a WSN of clustered nature can be attained by optimizing consumption of power between Cluster Heads (CHs) was addressed in (Tao and Krunz, 2010). To investigate this tradeoff between energy and communication, the analytical model incorporated both intra- and inter-cluster traffic (Tao and Krunz, 2010) using Rayleigh fading model for inter-cluster communications.

Two solutions (Suat and Çam, 2010) were thoroughly investigated for data collection. The author initially used scheduling of time using single frequency channel and transmission power control is combined to provide solutions related to interference, scheduling transmissions using multiple frequencies is proven to be of more efficient.

Most research works on relay selection observed channel conditions for decision making process involved during relay-selection decision for the subsequent frame. In (Wei et al., 2010), a finite-state Markov channels in the relay-selection problem is addressed to maximize the spectral efficiency, avoid error propagation and increase the overall network lifetime but power control was unaddressed.

The capacity of WSN requires significant data rates and throughput. In (Felemban et al., 2010) different benefits of combining the antennas into wireless sensor networks are presented using Sectored Antenna Medium Access Control (SAMAC), to fully utilize sectored antennas, is introduced.

In this study, Imbalanced Clustering Energy-Efficient (ICEE) mechanism for periodical data gathering organizes the network in an efficient manner via imbalanced clustering and multi hop routing. Simulation results show that ICEE successfully balances the energy consumption over the network and achieves a remarkable network lifetime improvement. The rest of this study is organized as follows: Section II covers related work in this area; Section III describes the periodical data assembly using imbalanced clustering energy efficient mechanism. Section IV presents with experimental results and $\mathrm{V}$ discuss about the simulation efforts using NS2 and the analysis of the results obtained with existing approach; section VI concludes paper.

\section{STATE-OF-THE ART}

Redundant Radix based Number (RBN) represent encoding and data transmission for different applications combines data to its equivalent redundant binary number representation (Koushik et al., 2011). Analysis does not consider ripple effect of symbol errors in RBN encoded data. The probability model to measure redundancy observed in path and redundancy of source level and the intrusion level under which the lifetime of a HWSN is maximized (Hamid and Chen, 2013). Min-max approximation approach measure area for tracking and solved using Semi Definite Programming (SDP) relaxation and deployed cubic function for navigating the mobile sensor (Enyang et al., 2013). Coverage-aware approach in camera-based wireless sensor networks is not explored. The image data's redundancy extracted from different cameras with views formed in overlapped manner, obtained using visual sensor networks (Stanislava and Wendi, 2009). Moreover, greedy and distributed types of algorithm were presented using simulation that results in high-quality placements for the additional sensors (Bredin et al., 2010).

Flocking Algorithm (FA) and a Particle Swarm Optimizer (PSO), technique develop a non convex mathematical model (Haijun et al., 2012). It is not implemented in more complex dynamic environments. Relay node placement under weaker connectivity requirement are not performed (Misra et al., 2010).

Hill Climbing key dissemination approach ensures nodes closer to data sources has stronger filtering capacity using data aggregation but dissemination protocols for wireless sensor networks are not addressed (Zhen and Guan, 2010). Hierarchical Heterogeneous Wireless Networks (HHWNs), model is based on the control framework at the physical layer designed using non convex mathematical model followed by organization and control of backbone nodes in HHWN. Finally, employ PSO, to optimize the HHWN by collecting global information from entire system (Zhang et al., 2012).

Secure and Distributed Reprogramming Protocol (SDRP) is vulnerable to an impersonation attack where adversary impersonates any authorized user. SDRP fails in integrating SDRP with a more efficient reprogramming protocol, leading to more secure and efficient distributed reprogramming (Daojing et al., 2013). Threshold Distributed Energy Efficient Clustering (TDEEC) protocol 
improves stability and energy efficient property of the heterogeneous wireless sensor network (Parul and Ajay, 2010). Cluster-tree repair algorithm associate node fails or moves. Real implementation of cluster generation algorithm using a test bed environment is not evaluated (Wang and Qian, 2012). Here, we extend this approach by defining periodical data assembly using imbalanced clustering energy efficient mechanism. Moreover, we now discuss to enhance the cluster efficiency and present considerably extended evaluation results, assessing not only to decide on the correct power level but also helps to create cluster of imbalanced size. In summary, our contributions are:

- To facilitate the nodes to decide on the correct power level to correspond with the base station

- To help to create clusters of imbalanced size

- To make nodes communicate directly with the base station in case of limited transmission range and

- Finally to use power control to send data to a farther receiver

\section{PERIODICAL DATA ASSEMBLY USING IMBALANCED CLUSTERING ENERGY EFFICIENT MECHANISM}

In this section, periodical data assembly using imbalanced clustering energy efficient mechanism is presented with the help of an architecture diagram followed by the system model with detail provided for imbalanced clustering algorithm.

During the network deployment stage, base station transmits message to all nodes at convinced power level. Comprehensive descriptions regarding imbalanced clustering algorithm is provided in the following subsections with the help of the architecture diagram.

Figure 1 gives an overview of the ICEE mechanism. The three steps involved in ICEE mechanism are elaborated as follows: The first step in ICEE performs the construction of sensor network. Here the circles of imbalanced size represent clusters whereas the traffic between cluster heads illustrates multi hop forwarding method.

Once the process of network construction gets accomplished, during second step, data are assembled periodically in a way that the cluster heads closer to the base station have lesser sizes and act as routers than the cluster heads farther from the base station during data delivery. The third and the final step in ICEE mechanism is imbalanced clustering and multi hop routing where cluster head election and partition node of imbalance size takes place. At this juncture, each cluster head spends its energy during the intracluster and inter-cluster which is proportional to the number of nodes surrounded by the cluster. Alternately, in the proposed ICEE, clustering algorithms results in the clusters of even size, with cluster heads consuming even amount of energy during the intra-cluster data processing phase. Finally, inter-process communication takes place.

\subsection{System Model}

A sensor network consisting of ' $M$ ' sensor nodes is considered which consistently positioned over a vast field to monitor the environment in an intermittent manner. Consider a network area corresponding to a set of sensor nodes $\mathrm{P}=\left\{\mathrm{p}_{1} ; \mathrm{p}_{2} \ldots \ldots \ldots \mathrm{p}_{\mathrm{M}}\right\}$ where $[\mathrm{P}]=\mathrm{M}$, where the $i^{\text {th }}$ sensor denotes by $p_{i}$.

Each node in ICEE is allocated with a unique Identifier (ID), where nodes needn't be connected with GPS competent unit. The nodes in ICEE use power control that are dissimilar and are at the same time proportionate with the quantity of transmission depending on the distance from the node to the receiver. The node in ICEE computes the approximate distance to make symmetric link based on the established signal power, if the transmitting power is given.

ICEE considers both the free space ( $l^{2}$ power loss) and the multi-path fading $\left(l^{4}\right.$ power loss) channel depends on distance between the transmitter and receiver. The energy consumed is given by:

$$
\begin{aligned}
& E C_{T x}(n, D)=E C_{\text {ele }}+n \in E C_{m p f} \\
& E C_{T x}(n, D)=E C_{\text {ele }}+n \in E C_{f s} l^{4}, l \geq l_{0}
\end{aligned}
$$

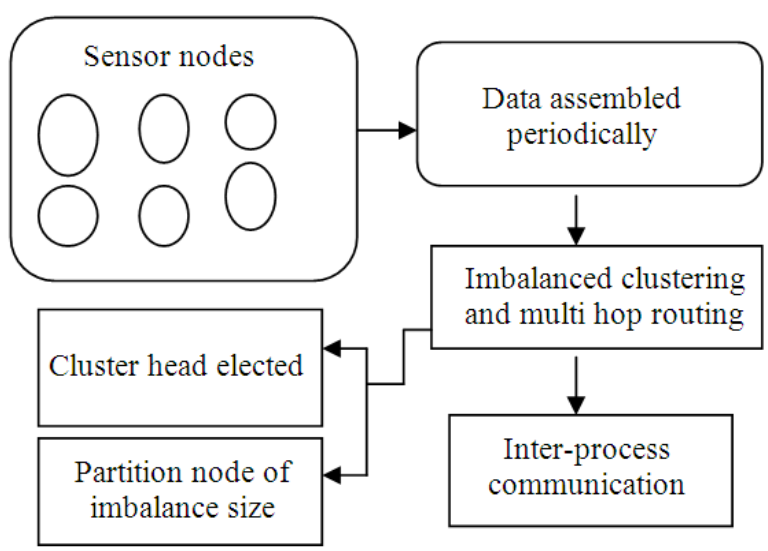

Fig. 1. Architecture diagram of ICEE mechanism 
where, $E C_{T x}$ denotes energy consumed during transmission of ' $n$-bit' packet over distance ' $D$ ' and ' $E C_{\text {ele }}$ ' sensor electronics transmission/reception signals with ' $E C_{m p f}$ ' representing energy consumed during multipath fading, ' $E C_{f s}$ ' representing the energy consumption for free space and to receive message, energy consumption is given by:

$$
R_{T x}(n)=n T_{\text {ele }}
$$

where, Equation 3 denotes energy consumption to receive the message of ' $n$-bit' packet ' $R_{T x}(n)$ '. A sensor node also consumes $D_{T x}$ amount of energy for data aggregation.

\subsection{Imbalanced Clustering Algorithm}

During data assembly in ICEE, based on energy consumption across the sensor network, selection of cluster head is performed. ICEE mechanism involves distributed cluster head competitive algorithm, where the selection of cluster head is based on the residual energy of each node.

During the first round, several uncertain cluster heads compete for final cluster heads. Each node results in uncertain cluster head with probability 'TP', a predefined threshold. Other nodes stay waiting for the selection process of cluster head to get completed. Suppose $\mathrm{p}_{\mathrm{i}}$ becomes an uncertain cluster head, with a competition range $\mathrm{Q}_{\text {comp }}$, represents the function of its distance from the node to the base station. The goal to be arrived in ICEE is that if $p_{i}$ becomes a cluster head at the end of the competition; there will no cluster head other than $p_{i}$.

Figure 2 illustrates the topology of uncertain cluster heads, where circles denote varying competition ranges of uncertain cluster heads. In Fig. 2 , let $\mathrm{p}_{1}$ and $\mathrm{p}_{2}$ both denote the cluster heads, whereas $\mathrm{p}_{3}$ and $\mathrm{p}_{4}$ cannot be the cluster head. In ICEE, higher the number of clusters is closer to each other in a network; maximum is the diminishing radius from the cluster head to the base station as the distance to the Base Station (BS) reduces with maintaining radius in the network. The algorithm for an arbitrary node $\mathrm{p}_{\mathrm{i}}$ is shown in upcoming section.

Each uncertain cluster head maintains a set $\mathrm{P}_{\mathrm{CH}}$ of its adjacent uncertain cluster heads, uncertain head $p_{j}$ is an adjacent node of $p_{i}$ if $p_{j}$ is in $p_{i}$ 's competition diameter or $p_{i}$ is in $p_{j}$ 's competition diameter. Whether an uncertain cluster head $\mathrm{p}_{\mathrm{i}}$ become a final cluster head depends on the nodes in $p_{i}, P_{C H}$ only, i.e., the algorithm is distributed. In the cluster head selecting algorithm, the broadcast radius of every control message is $Q_{\text {comp }}^{0}$ 'thus $p_{i}$ hear all messages from node in its $\mathrm{P}_{\mathrm{CH}}$.

\section{// ICEE cluster head Selection}

$1: \mu \leftarrow \operatorname{RAND}(0,1)$

2: If $\mu<$ TP then beuncertainhead $\leftarrow$ TRUE

3: End If

4: If beuncertainhead = TRUE ParticipateHeadMsg (ID; $\left.Q_{\text {comp }} ; \mathrm{QE}\right)$ Else End if

5: On receiving a ParticipateHeadMsg form node $p_{j}$

6: If $l\left(p_{i}, p_{j}\right)<p_{j} Q_{\text {comp }} \operatorname{OR} l\left(p_{i}, p_{j}\right)<p_{i} \cdot Q_{\text {comp }}$ Add $p_{j}$ to $p_{i \text {. }}$ $p_{C H}$ End if

7: While beuncertainhead $=$ TRUE do

8: If $p_{i}: Q D>p_{j}: Q D, £ p_{j} € p_{i} . P_{C H}$ then FinalHeadMsg (ID) else End if

9: If $p_{j} € p_{i} \cdot P_{C H}$ then QuitElectionMsg (ID) else End if

10: If $p_{j} € p_{i} . P_{C H}$ then Remove $p_{j} . P_{C H}$ from

11: End If

12: End While

\subsection{ICEE Multi-Hop Routing}

The cluster heads transport data to the base station, each cluster head first assemble the data from its cluster members and then sends the packet to the base station via multi-hop communication. Threshold TP_MAX in multi-hop onward model defines the node's distance to the base station. If the distance is smaller than TP_MAX, it transmits its data to the base station directly; otherwise it find a relay node which forwards its data to the base station. In case of existing scheme, cluster head $p_{i}$ chooses a node to forwarding its data from its candidate set $Q_{C H}$, which is defined as Equation 4:

$$
p_{i} Q_{C H}=\left\{p_{j} \mid\left(p_{i}, p_{j}\right) \leq k p_{i .} Q_{\text {comp }}, l\left(p_{j} B S\right)<l\left(p_{j}, B S\right)\right\}
$$

where, $\mathrm{k}$ is the minimum integer that let $p_{i}, Q_{C H}$ contains at least one item. To decrease wireless channel interference, ICEEE improved to choose an adjoining node as the relay node. According to Equation 1 to 3 in ICEE mechanism, defined as Equation 5:

$$
l_{\text {relay }}^{2}=l^{2}\left(p_{i}, p_{j}\right)+l^{2}\left(p_{j}, B S\right)
$$




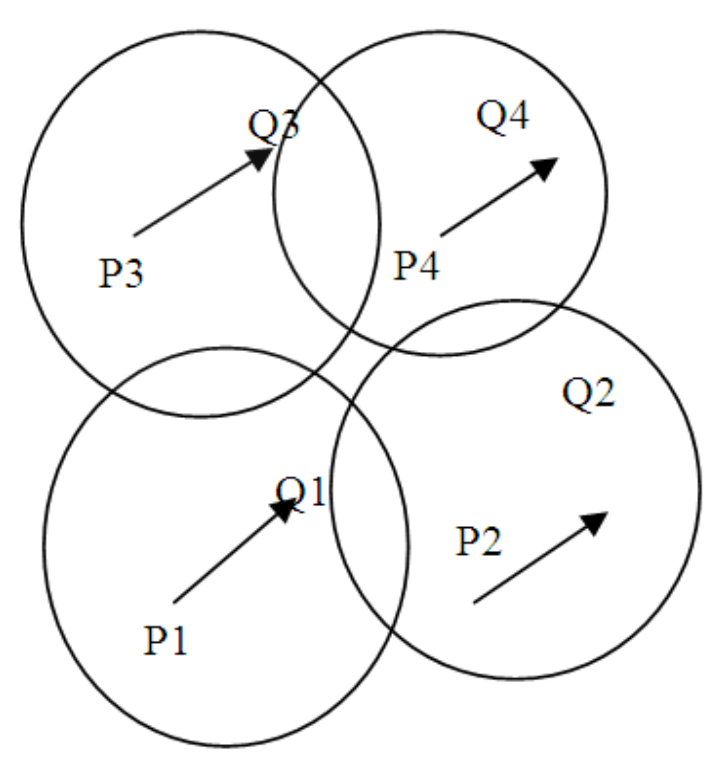

Fig. 2. Uncertain cluster head competition

The bigger the $l^{2}$ relay is the more energy will be added to the ICEE relay process. In ICEE mechanism, $p_{i}$ chooses $p_{j}$ with more residual energy from the two smallest $l^{2}$ relay nodes in $p_{i}, Q_{C H}$ as its relay node and finally a tree rooted at the base station is constructed.

\section{EXPERIMENTAL RESULTS}

The performance of ICEE is measured using ns2 simulations. The experimental results of ICEE mechanism is measured in terms of power consumption, communication energy and cluster probability ratio in wireless sensor network. The simulation settings is run over time period of $800 \mathrm{sec}$ with a field size of $500 \mathrm{~m} \mathrm{x}$ $500 \mathrm{~m}$ at the speed of $60 \mathrm{~m} \mathrm{sec}^{-1}$.

Communication energy in ICEE measures amount of energy consumed to transfer data from source to destination, a measured of Joules $(\mathrm{J})$. The rate of power consumed in ICEE mechanism is evaluated to identify the consumption of power that performs the operational task and this is measured in terms of Watt (W). Cluster head probability ratio in ICEE is defined as the probability to choose the cluster head to group similar elements gathered closely together. The cluster head based ICEE mechanism improves the probability ratio by transmitting the periodic data in wireless sensor network.

\section{DISCUSSION}

The experimental results provided in the prior section demonstrated the lower communication energy, power consumption and cluster head probability ratio with the help of ICEE mechanism. In this section, detailed discussion is presented during the simulation process of ICEE. ICEE performs effective periodical data assembly with minimal communication energy and power consumption. The performance of the Imbalanced Clustering Energy-Efficient (ICEE) mechanism is compared with the Cooperative Networking protocol (CONET) (Yoo and Park, 2011) protocol in WSN. Fig. 3 describe the communication energy based on user request and compared with the CONET (Yoo and Park, 2011) in an elaborate manner. The communication energy as illustrated is comparatively lower than the existing CONET (Yoo and Park, 2011) because the energy spent using ICEE mechanism depends on $\mathrm{C}_{\mathrm{Tx}}$ with the transmission of ' $\mathrm{n}$ ' bit packet over distance ' $D$ ' and broadcasted also based on electronics transmission signals. As the clustering size increases, the energy used for communication also increases, but compared to CONET protocol, communication energy using ICEE mechanism is decreased by $2-5 \%$.

Figure 4 describes the power consumption of the sensor nodes in wireless network and comparison made with CONET (Yoo and Park, 2011) protocol. From the figure it is evident that the power consumption using ICEE is less because the nodes in the network computes approximate distance to make a symmetric link based on the established signal power and use the power control with different quantity of transmission power. Finally, ICEE mechanism reduces $5-10 \%$ of power consumption when compared with the CONET protocol.

Figure 5 describes cluster head probability ratio based on the clustering node and comparison is made with the existing CONET (Yoo and Park, 2011) protocol. The cluster head collection in ICEE is based on the residual energy of each node which improves the probability ratio by $15-20 \%$ when compared to the CONET (Yoo and Park, 2011) protocol. Finally, a small interval between the time the first node die and the time the last node die implies that ICEE mechanism has successfully solved the hot spots problem and performs the effective periodical data assembly. 
Munusamy, K. and R.M.S. Parvathi / Journal of Computer Science 10 (10): 2047-2054, 2014

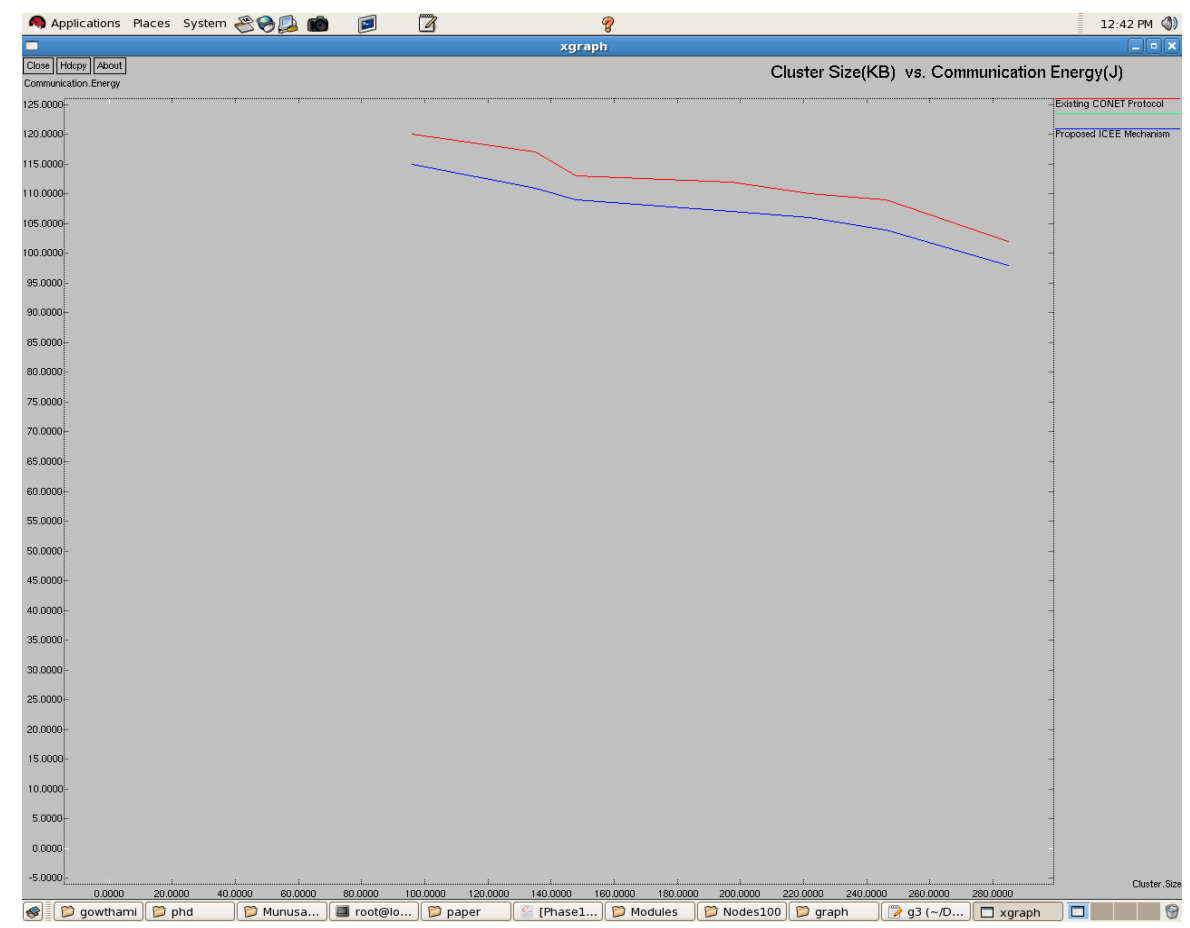

Fig. 3. Cluster size Vs. communication energy

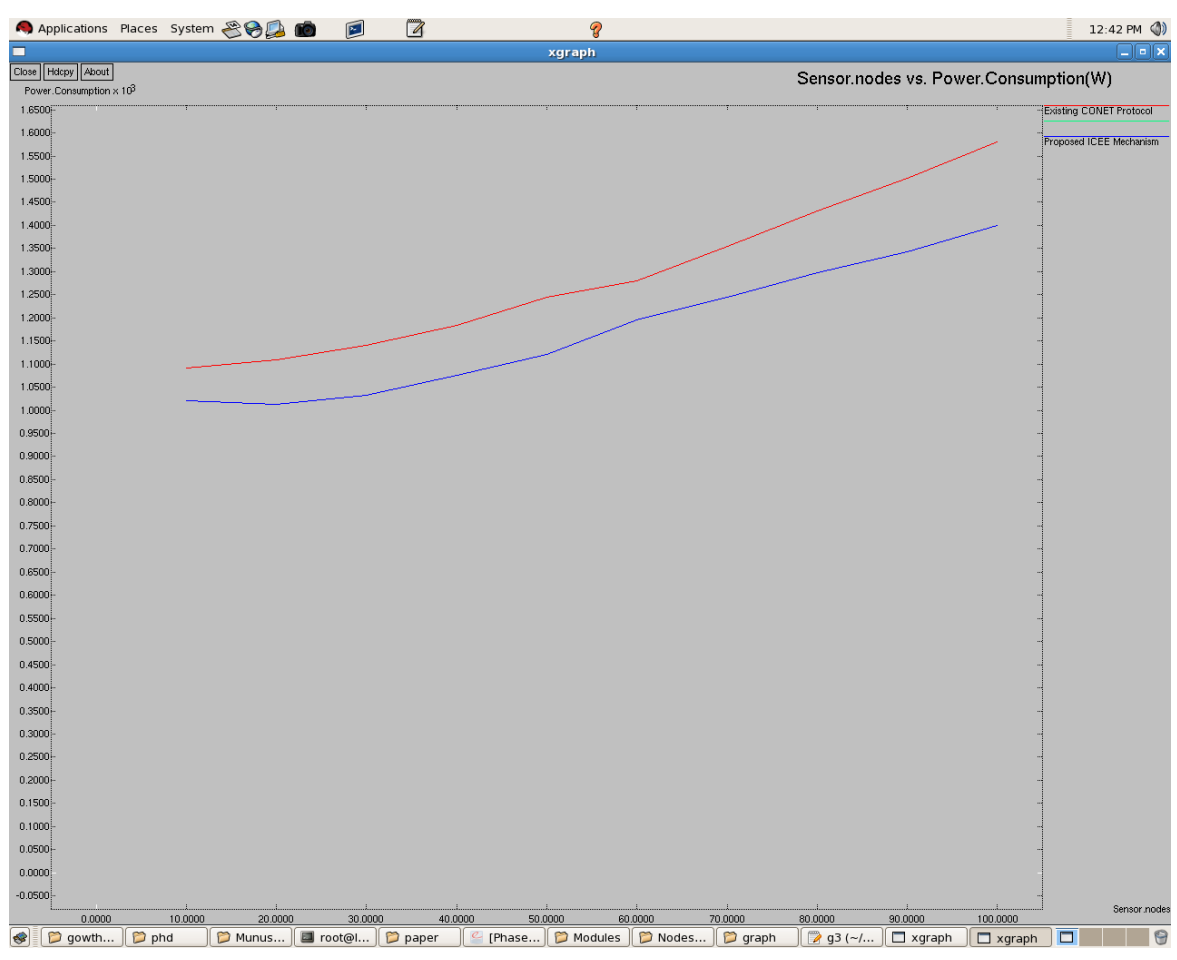

Fig. 4. No. of sensor nodes Vs. power consumption 


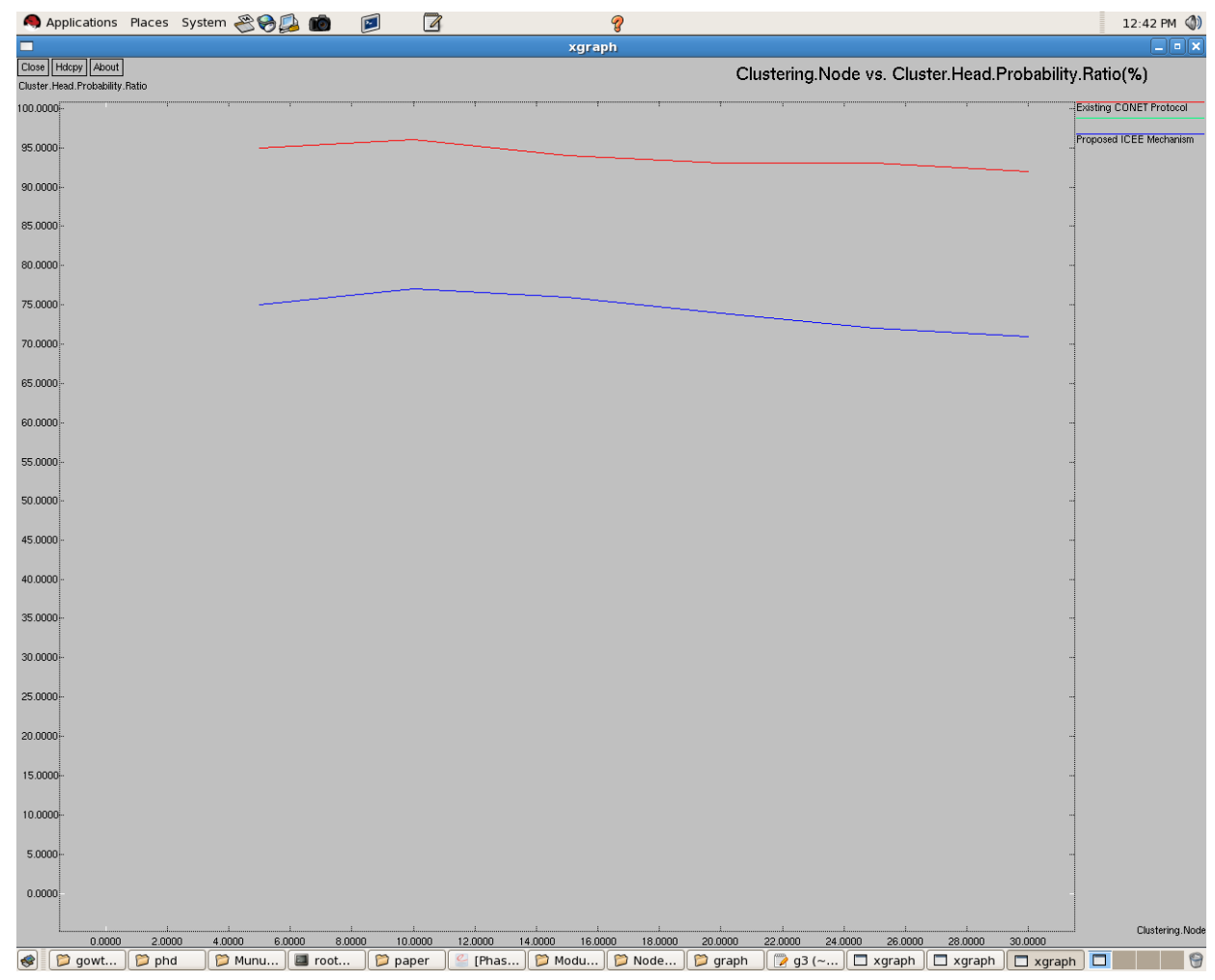

Fig. 5. Clustering node Vs. cluster head probability ratio

\section{CONCLUSION}

In this study, the problem of periodic data assembly in wireless sensor networks using Imbalanced Clustering Energy-Efficient mechanism in wireless sensor networks is presented. It organizes the network via imbalanced clustering and multi-hop routing with the cluster heads being elected using localized competition. ICEE partitions the nodes into clusters of imbalanced size and clusters closer to the base station have lesser sizes than those farther away from the base station. The results presented attain high energy efficiency and amplify the network scalability. Finally, it minimizes $4.57 \%$ energy of sensor nodes in WSN. Experiments are conducted using NS2 and the simulation result of ICEE outperforms the CONET protocol in terms of communication energy, power consumption and cluster head probability ratio in wireless sensor network. An error-free communication links are assumed which though not possible in practical scenarios can be applied to relay network level which is outside the scope of the work and left for future work.

\section{REFERENCES}

Bredin, J.L., E.D. Demaine, M.T. Hajiaghayi and R. Daniela, 2010. Deploying sensor networks with guaranteed fault tolerance. IEEE/ACM Trans. Network., 18: 216-228. DOI: 10.1109/TNET.2009.2024941

Daojing, H., C. Chun, S. Chan, J. Bu and L.T. Yang, 2013. Security analysis and improvement of a secure and distributed reprogramming protocol for wireless sensor networks. IEEE Trans. Indust. Electron., 60: 5348-5354. DOI: 10.1109/TIE.2012.2218562

Enyang, X., D. Zhi and S. Dasgupta, 2013. Target tracking and mobile sensor navigation in wireless sensor networks. IEEE Trans. Mobile Comput., 12: 177-186. DOI: 10.1109/TMC.2011.262

Felemban, E., S. Vural, R. Murawski, E. Ekici and K. Lee et al., 2010. SAMAC: A cross-layer communication protocol for sensor networks with sectored antennas. IEEE Trans. Mobile Comput., 9: 1072-1088. DOI: 10.1109/TMC.2010.61 
Haijun, Z., L. Jaime, C.C. Davis and S.D. Milner, 2012. Nature-inspired self-organization, control and optimization in heterogeneous wireless networks. IEEE Trans. Mobile Comput., 11: 12071222.

Hamid, A.H. and I.R. Chen, 2013. Redundancy management of multipath routing for intrusion tolerance in heterogeneous wireless sensor networks. IEEE Trans. Netw. Service Manage., 10: 189-203. DOI: 10.1109/TNSM.2013.043013.120282

Kim, J., X. Lin, N.B. Shroff and P. Sinha, 2010. Minimizing delay and maximizing lifetime for wireless sensor networks with anycast. IEEE/ACM Trans. Network., 18: 515-528. DOI: 10.1109/TNET.2009.2032294

Koushik, S., P.S. Bhabani and D. Debasish, 2011. An energy-efficient communication scheme for wireless networks: A redundant radix-based approach. IEEE Trans. Wirel. Commun., 10: 550-559. DOI: 10.1109/TWC.2010.120610.100244

Misra, S., S.D. Hong, G. Xue and J. Tang, 2010. Constrained relay node placement in wireless sensor networks: Formulation and approximations. IEEE/ACM Trans. Network., 18: 434-447. DOI: 10.1109/TNET.2009.2033273

Parul, S. and K.S. Ajay, 2010. Energy efficient scheme for clustering protocol prolonging the lifetime of heterogeneous wireless sensor networks. Int. J. Comput. Applic., 6: 30-36.

Rocio, A.V., A.G. Marques and C.S. Jesus, 2011. Optimal selective forwarding for energy saving in wireless sensor networks. IEEE Trans. Wirel. Commun., 10 : 164-175. DOI: 10.1109/TWC.2010.102810.100014
Stanislava, S. and B.H. Wendi, 2009. Cluster head election techniques for coverage preservation in wireless sensor networks. Ad Hoc Netw., 7: 955972. DOI: 10.1016/j.adhoc.2008.08.006

Suat, O. and H. Çam, 2010. Integration of false data detection with data aggregation and confidential transmission in wireless sensor networks. IEEE/ACM Trans. Network., 18: 736-749. DOI: 10.1109/TNET.2009.2032910

Tao, S. and M. Krunz, 2010. Coverage-time optimization for clustered wireless sensor networks: A powerbalancing approach. IEEE/ACM Trans. Network., 18: 202-215. DOI: 10.1109/TNET.2009.2022936

Wang, X. and H. Qian, 2012. Constructing a 6lowpan wireless sensor network based on a cluster tree. IEEE Trans. Vehicular Technol., 61: 1398-1405. DOI: 10.1109/TVT.2012.2185260

Wei, Y., F.R. Yu and M. Song, 2010. Distributed optimal relay selection in wireless cooperative networks with finite-state markov channels. IEEE Trans. Vehicular Technol., 59: 2149-2158. DOI: 10.1109/TVT.2010.2041803

Yoo, J.W. and K.H. Park, 2011. A cooperative clustering protocol for energy saving of mobile devices with WLAN and bluetooth interfaces. IEEE Trans. Mobile Comput., 10: 491-504. DOI: 10.1109/TMC.2010.161

Zhang, H., J. Llorca, C.C. Davis and S.D. Milner, 2012. Nature-inspired self-organization, control and optimization in heterogeneous wireless networks. IEEE Trans. Mobile Computing, 11: 1207-1222.

Zhen, Y. and Y. Guan, 2010. A dynamic en-route filtering scheme for data reporting in wireless sensor networks. IEEE/ACM Trans. Network., 18: 150163. DOI: 10.1109/TNET.2009.2026901 